\title{
Sputum signatures for invasive pulmonary aspergillosis in patients with underlying respiratory diseases (SPARED): study protocol for a prospective diagnostic trial
}

Wei Xiao ${ }^{1 \dagger}$, De-ying Gong ${ }^{2 \dagger}$, Bing Mao ${ }^{1}$, Xin-miao Du ${ }^{3}$, Lin-Li Cai ${ }^{1}$, Min-yu Wang ${ }^{1}$ and Juan-juan Fu ${ }^{1 *}$ (D)

\begin{abstract}
Background: Invasive pulmonary aspergillosis (IPA) has been increasingly reported in patients with underlying respiratory diseases (URD). Early diagnosis of IPA is crucial for mortality reduction and improved prognosis, yet remains difficult. Existing diagnostic tools for IPA largely rely on the detection of biomarkers based on serum or bronchoalveolar lavage fluid (BALF), both of which have their limitations. The use of sputum sample is non-invasive, and Aspergillus detection is feasible; however, the usefulness of sputum biomarkers for the diagnosis of IPA, especially in patients with URD, has not been systematically studied.

Methods: This is a prospective diagnostic trial. At least 118 participants will be recruited from respiratory wards and intensive care units. IPA is defined according to the EORTC/MSG criteria modified for patients with URD. Induced sputum and blood will be collected, and BALF will be obtained by bronchoscopy. Sputum biomarkers, including galactomannan, Aspergillus DNA, triacetylfusarinine and bis(methylthio)gliotoxin will be determined, and the presence of a JF5 antigen will be examined with a lateral fluid device. The sensitivity, specificity, negative predictive value, positive predictive value and diagnostic odds ratio will be computed for different biomarkers and compared using the McNemar $x^{2}$ test. Receiver operating characteristic analyses will be performed, and the cut-off values will be established. Participants will receive follow-up evaluations at 3 months and 6 months after recruitment. The difference in hospital stay and survival will be analysed, and the relationships between the levels of biomarkers and hospital stay and survival will be analysed via regression models.
\end{abstract}

Discussion: We have developed and verified the feasibility of Aspergillus-related biomarker assays for sputum. The study findings will contribute to a novel look at the diagnostic performance of sputum biomarkers in IPA and provide important insight into the improvement of the early diagnosis of IPA, particularly in patients with URD.

Trial registration: This study has been registered with the Chinese Clinical Trial Registry (ChiCTR-DPD-16009070) on 24th of August 2016.

Keywords: Invasive pulmonary aspergillosis, Underlying respiratory diseases, Diagnosis, Biomarker, Galactomannan, Lateral flow device, Real-time PCR, Triacetylfusarinine, Bis(methylthio)gliotoxin

\footnotetext{
* Correspondence: juanjuan.fu@outlook.com

${ }^{\dagger}$ Wei Xiao and De-ying Gong contributed equally to this work.

${ }^{1}$ Respiratory Group, Department of Integrated Traditional Chinese and

Western Medicine, West China Hospital, Sichuan University, Chengdu, China

Full list of author information is available at the end of the article
}

(c) The Author(s). 2018 Open Access This article is distributed under the terms of the Creative Commons Attribution 4.0 International License (http://creativecommons.org/licenses/by/4.0/), which permits unrestricted use, distribution, and reproduction in any medium, provided you give appropriate credit to the original author(s) and the source, provide a link to the Creative Commons license, and indicate if changes were made. The Creative Commons Public Domain Dedication waiver (http://creativecommons.org/publicdomain/zero/1.0/) applies to the data made available in this article, unless otherwise stated. 


\section{Background}

Invasive pulmonary aspergillosis (IPA) is a fungal infection that occurs primarily in patients with severe immunodeficiency [1]. In recent years, IPA has been increasingly recognized in patients without haematologic malignancy or organ transplantation [2-9], especially in those with underlying respiratory diseases (URD) $[2-5,10-12]$. Remarkably, the mortality rate among non-neutropenic patients was significantly higher than that in neutropenic patients [12], such that a mortality rate of greater than $90 \%$ in patients with chronic obstructive pulmonary disease was reported [13].

Although a favourable clinical outcome of patients with IPA is largely influenced by the initiation of effective antifungal treatment [14], the early diagnosis of IPA is still notoriously difficult, and few diagnostic tools are available [15]. The "gold standard" to confirm the diagnosis is the histopathological examination of lung tissue obtained by thoracoscopic or open-lung biopsy [16]. Considering its limitation in terms of feasibility and sensitivity in clinical practice, the European Organization for Research and Treatment of Cancer/Mycoses Study Group (EORTC/MSG) proposed diagnostic criteria for IPA that integrate host factors, clinical evidence and mycological findings [17]. However, the diagnosis of IPA in patients with URD remains challenging. The host factors defined by the EORTC/MSG framework do not necessarily apply to patients in the respiratory wards or intensive care units [2], and the official group recognized this as "an omission" [17].

A variety of sample types and biomarkers have been explored and validated in the journey to seek mycological evidence for the diagnosis of IPA, and different methodologies and matching signatures have been investigated (Fig. 1). Direct mycological proof by culture is rarely feasible due to the low sensitivity and long turnaround time $[18,19]$. Therefore, indirect tests that detect Aspergillus-related biomarkers, e.g., galactomannan (GM), in body fluids were developed, showed improved diagnostic performance and were included in the EORTC/MSG framework [17]. Serum biomarkers, in particular, Aspergillus DNA (A-DNA) and GM, have been intensively studied in haematological patients in the past two decades which exhibited good performance $[20,21]$. However, these tests had lower sensitivity when applied in non-haematological patients [22, 23], probably because non-neutropenic patients tend to develop airway invasive aspergillosis instead of angioinvasive aspergillosis [24, 25]. This further indicates that biomarker detection based on organ specific samples may be associated with better diagnostic performance for IPA in non-neutropenic patients. Indeed, biomarker detection using bronchoalveolar lavage fluid (BALF) has been found to be associated with a higher diagnostic accuracy for IPA compared to serum in these patients $[6-8,26]$. However, bronchoscopy is an invasive procedure and is not feasible for all patients in clinical practice. In contrast, induced sputum sampling is non-invasive, convenient and low cost and, if successful, often precludes the need for bronchoscopy. Sputum is expectorated from local sites of infection and can be used for microbiologic examinations, therefore, it has the potential to be used for biomarker determination in IPA.

We searched the literature using the terms "sputum", "sputa", "bronchial secretion", "bronchial aspirate", "respiratory sample" and "aspergillosis", "Aspergillus" in Medline, EMBASE and PubMed databases up to the 20th of May 2017. Seven studies that assessed the GM assay with sputum samples were identified (Table 1), of

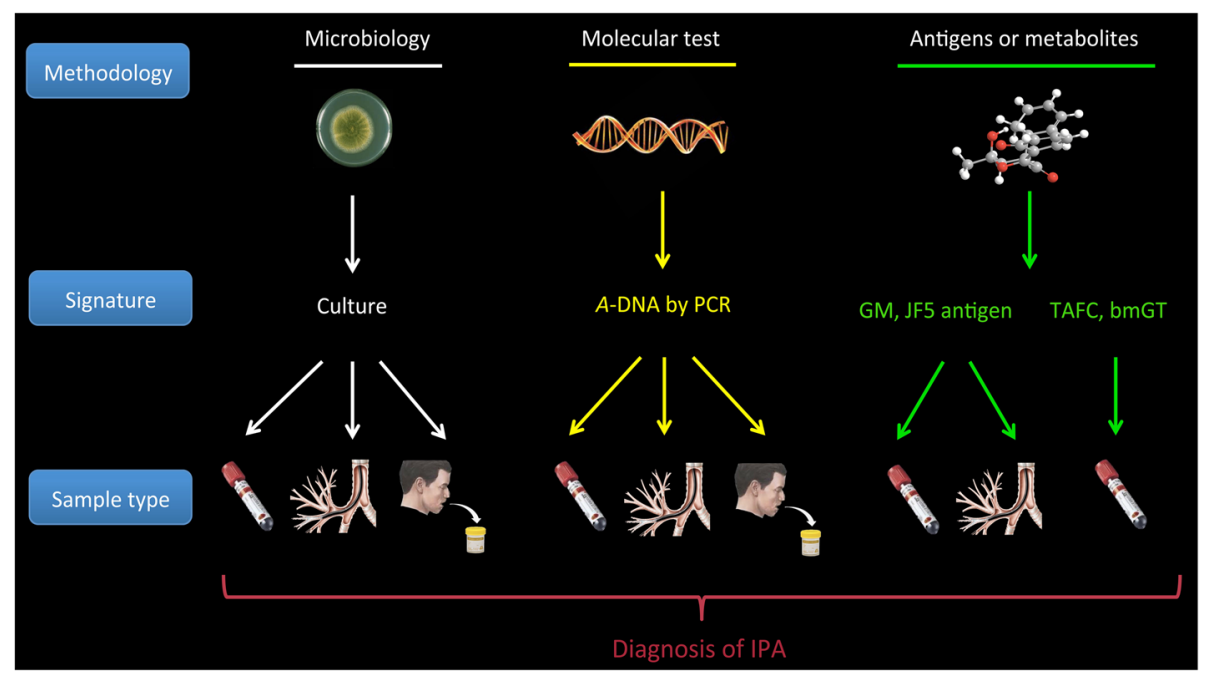

Fig. 1 Current biomarkers for the diagnosis of IPA using different sample types. Abbreviations: A-DNA = aspergillus DNA; bmGT = bis(methylthio)gliotoxin; $\mathrm{GM}$ = galactomannan; $\mathrm{PCR}=$ polymerase chain reaction; TAFC = triacetylfusarinine 
Table 1 Studies assessing sputum GM assay for various Aspergillus events

\begin{tabular}{lllll}
\hline Author & Year & Aspergillus events & Underlying diseases & Sputum type \\
\hline Kimura [27] & 2009 & IPA & HD & spontaneous/induced \\
Fraile [32] & 2012 & IPA $^{\text {a }}$ & not classified & not reported \\
Delfino [34] & AC & cystic fibrosis & not reported \\
Baxter [29] & 2012 & AC & cystic fibrosis & spontaneous \\
Baxter [28] & 2013 & ABPA & cystic fibrosis & spontaneous \\
Chanzá [33] & 2014 & IPA $^{\text {a }}$ & HD and NHD & not reported \\
Dhillon [35] & ABPA & cystic fibrosis & not reported \\
\hline
\end{tabular}

Abbreviations, ABPA allergic bronchopulmonary aspergillosis, AC Aspergillus colonization, $H D$ haematological disease, IPA invasive pulmonary aspergillosis, NHD non-haematological disease

${ }^{a}$ EORTC/MSG diagnostic criteria were not used.

${ }^{\mathrm{b}}$ Patients recruited from Infectious, Pneumology, Oncology Services and Critical Care Unit, without specific underlying diseases classified.

which only the study by Kimura et al. [27] tested the efficacy of sputum GM for the diagnosis of IPA in haematological patients in a well-designed manner. The results of this study identified a sensitivity of $100 \%$ and a specificity of $62.2 \%$ for sputum GM at the cut-off value of 1.2 optical density index (ODI). However, the small sample size impeded the external validity of the study and the validation of an optimal cut-off value. Some studies have assessed $A$-DNA in sputum via the polymerase chain reaction (PCR) assay; however, these studies exclusively aimed at identifying the existence of Aspergillus in sputum rather than assessing the diagnostic performance for IPA [28-31]. Fraile et al. [32] and Chanzá et al. [33] intended to evaluate the sputum PCR assay for the diagnosis of IPA; however, diagnostic criteria according to the EORTC/MSG framework were not applied. Our study will use real-time PCR (RT-PCR) to detect sputum $A$-DNA for IPA in patients with URD diagnosed by the EORTC/MSG criteria.

Several other biomarkers that either target Aspergillus antigens or Aspergillus-related metabolites have been proposed for the diagnosis of IPA in recent years (Fig. 1) by a systematic literature review. Thornton et al. [36] developed a lateral flow device (LFD) that could be used at bedside to detect the JF5 antigen, an Aspergillus glycoprotein secreted during active fungal growth, which showed better diagnostic performance than GM in serum and BALF. Triacetylfusarinine (TAFC) and bis(methylthio)gliotoxin (bmGT) are toxic metabolites of Aspergillus and have been assayed by liquid chromatography tandem mass spectrometry in serum of haematology patients [37] or neutropenic patients, respectively. The above Aspergillus-related signatures present appropriate diagnostic accuracy for the diagnosis of IPA, yet have not been assessed in sputum samples or in patients with URD.

A study exploring the usefulness and diagnostic performance of sputum biomarkers for IPA in patients with URD is absent. Considering the incidence and high mortality of these patients and the great potential of using a sputum sample for Aspergillus detection, we will conduct this study to examine a broad spectrum of approaches and biomarkers related to Aspergillus detection using sputum samples from patients with URD to investigate the diagnostic performance of these sputum signatures for IPA in this study population. This study may help provide novel and useful diagnostic modalities, stepping over the existing obstacles to an early diagnosis of IPA in clinical practice.

\section{Methods/design \\ Study design}

This is a prospective diagnostic trial. The study protocol has been registered with the Chinese Clinical Trial Registry (ChiCTR-DPD-16009070). Participants will be enrolled from respiratory wards and intensive care units in the West China Hospital, Sichuan University of China. Inclusion criteria are patients (1) with URD including chronic pulmonary diseases (COPD, asthma, lung cancer, bronchiectasis, interstitial lung diseases, and cystic fibrosis) and acute pulmonary diseases (bacterial pneumonia, viral pneumonia, and acute bronchitis); (2) willing to provide informed consent; and (3) able to tolerate sputum induction. Exclusion criteria are patients (1) with underlying haematological malignancies; or (2) that are previous receipts of solid organ transplant; or (3) that are unable to produce sputum.

\section{Diagnostic criteria}

The diagnostic criteria of IPA are based on the 2008 EORTC/MSG framework [17]. Host factors, clinical criteria and mycological criteria to establish the diagnosis of IPA in this study population are listed in Table 2. In accordance with the study by Prattes et al. [11], URD is added as one of the host factors. We propose to make this modification due to several reasons. Host factors of the diagnostic criteria in previous guidelines were originally defined for haematological patients but have not been validated in non-neutropenic patients. Additionally, those typical host factors promoted by EORTC/MSG are frequently absent in non-haematological patients [2, 23]. Patients will be classified into proven, probable, possible 
Table 2 The diagnostic framework for IPA in patients with URD

\begin{tabular}{ll}
\hline Host factors & Recent history of neutropenia $\left(<0.5 \times 10^{9}\right.$ neutrophils $/ \mathrm{L}$ for $>10$ days) temporally related to the onset of infection \\
& Prolonged use of corticosteroids at a mean minimum dose of $0.3 \mathrm{mg} / \mathrm{kg} /$ day of prednisone equivalent for $>3$ weeks \\
& Treatment with immunosuppressants during the past 90 days \\
& Inherited severe immunodeficiency \\
& Underlying respiratory diseases including chronic pulmonary diseases (COPD, asthma, lung cancer, bronchiectasis, interstitial lung \\
& diseases, and cystic fibrosis) and acute pulmonary diseases (bacterial pneumonia, viral pneumonia, and acute bronchitis) \\
Clinical criteria & The presence of 1 of the following 3 signs on chest computed tomography: \\
& Dense, well circumscribed lesion(s) with or without a halo sign \\
& Air-crescent sign \\
& Cavity on chest computed tomography \\
Mycological & Cytologic or microscopic evidence of Aspergillus elements or recovery of Aspergillus in sputum or BALF \\
criteria & Positive GM test in serum or BALF
\end{tabular}

and no IPA groups according to the revised 2008 EORTC/MSG criteria [17] (Table 3).

\section{Participants screening and recruitment}

The study flowchart is shown in Fig. 2. Patients with respiratory diseases admitted into the hospital will be screened for inclusion and exclusion criteria for recruitment. The recruited patients will be subsequently assessed according to the clinical criteria shown in Table 2 , namely newly-developed abnormalities in CT scan associated with IPA, for the classification of no IPA or suspected IPA groups. Patients without clinical criteria will be identified as no IPA. For patients with typical clinical criteria, those presenting histopathological or cytopathological evidence of Aspergillus hyphae from needle aspiration or biopsy specimen, or positive culture result for Aspergillus from a sample obtained by sterile procedure from the lung will be identified as proven IPA. If lung biopsy is not performed or negative result is generated, mycological examinations will be performed including sputum/BALF culture or serum/BALF GM tests to further classify patients as probable or possible IPA. Sample collection and testing will be required to be performed prior to antifungal treatment.

\section{Sputum induction and sample processing}

Sputum induction using hypertonic saline (4.5\%) and spirometry (KoKo PD Instrumentation, Louisville, KY) will be performed as previously described [38]. 4.5\% saline will be inhaled from an ultrasonic nebulizer for doubling time periods (from $30 \mathrm{~s}$ to $4 \mathrm{mins}$ ), and a fixed cumulative sputum induction time of $15 \mathrm{~min}$ will be used for all participants.

Fungal culture will be performed on $100 \mu \mathrm{L}$ of sputum plugs carefully separated from saliva and inoculated directly onto potato dextrose agar [39]. The plates will be sealed and incubated for 7 days at $37^{\circ} \mathrm{C}$. After 7 days, Aspergillus will be identified based on macroscopic and microscopic features.

A minimum volume of $0.5 \mathrm{~mL}$ of sputum plugs will be pipetted into a sterile falcon centrifuge tube, and 8 volumes of phosphate buffer solution will be added, vortexed and incubated at room temperature for 30 mins, and $1.5 \mathrm{~mL}$ of the above sputum suspension will be stored for $A$-DNA extraction and RT-PCR assay.

A total of $200 \mu \mathrm{L}$ of filtered suspension will be used for quality assessment as previously described [38]. Briefly, in an adequate number of cells, the presence of pulmonary macrophages and the proportion of squamous cells are examined. A total cell count of leukocytes and cell viability is performed, and samples with a squamous cell percentage greater than $50 \%$ or viability less than $40 \%$ are excluded. The remainder of the sample will go through a sterile filter (Merk Millipore Ltd., Ireland) and will be stored in aliquots at $-80{ }^{\circ} \mathrm{C}$ for subsequent GM, LFD, TAFC and bmGT detection.

\section{BALF collection and sampling}

Fibreoptic bronchoscopy with bronchoalveolar lavage is performed in accordance with the American Thoracic Society guidelines [40]. Briefly, the bronchoscope will be placed in a wedge position within the selected bronchopulmonary segment. A total volume of $40 \mathrm{~mL}$ of normal

Table 3 IPA classification according to revised 2008 EORTC/MSG criteria

\begin{tabular}{ll}
\hline Proven IPA & $\begin{array}{l}\text { Histopathological or cytopathological examination of lung tissue showing Aspergillus hyphae from needle aspiration or biopsy } \\
\text { specimen with evidence of associated tissue damage, OR positive culture result for Aspergillus from a sample obtained by sterile } \\
\text { procedure from the lung. }\end{array}$ \\
Probable IPA & Host factors, AND clinical criteria, AND mycological criteria \\
Possible IPA & Host factors AND clinical criteria \\
No IPA & Other cases excluded from proven, probable and possible IPA groups \\
\hline
\end{tabular}




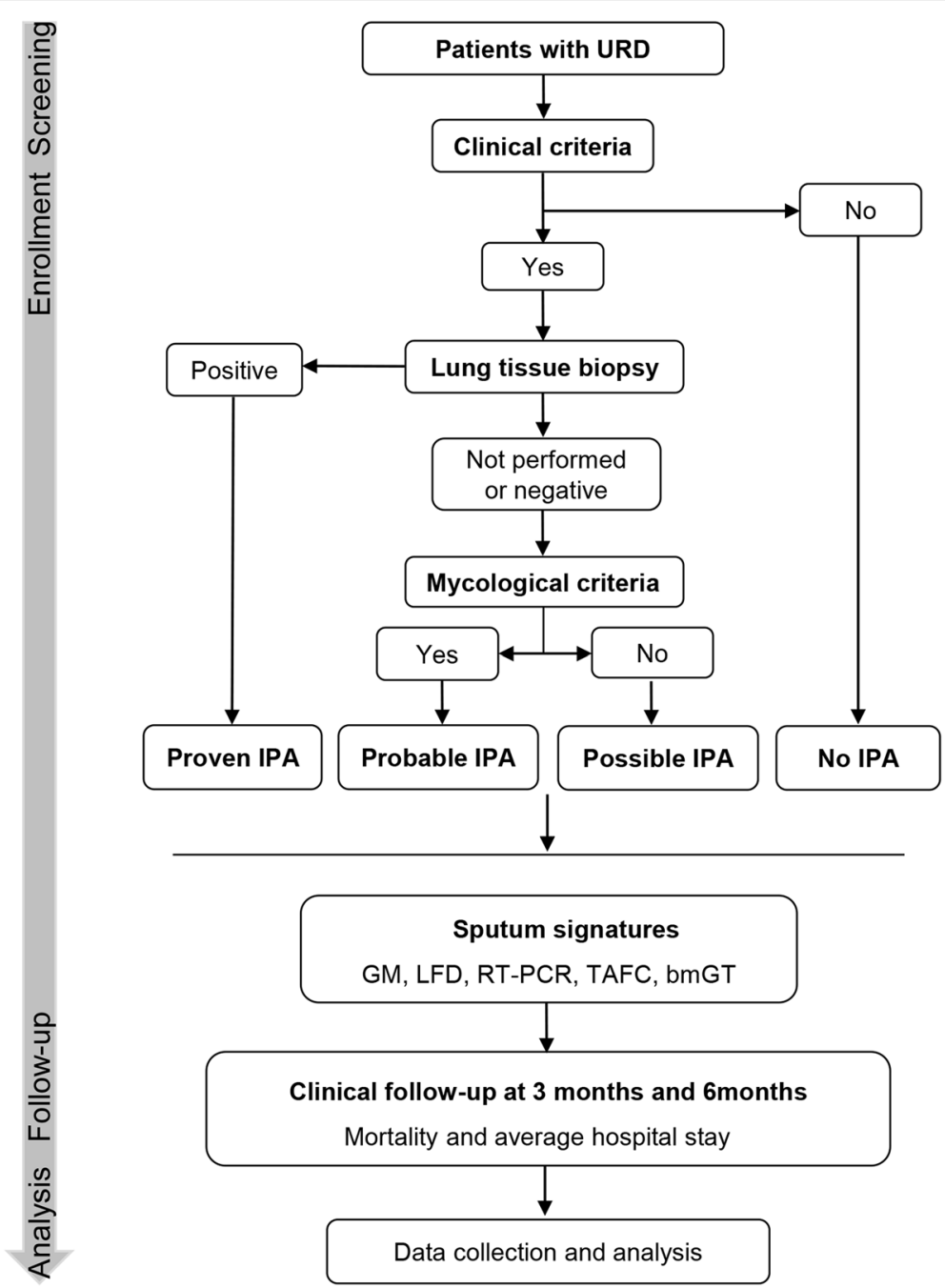

Fig. 2 Study flowchart. Abbreviations: bmGT = bis(methylthio)gliotoxin; BALF = bronchoalveolar lavage fluid; GM= galactomannan; IPA = invasive pulmonary aspergillosis; LFD = lateral flow device; RT-PCR = real time polymerase chain reaction; TAFC = triacetylfusarinine

saline (at room temperature) will be divided into two aliquots and instilled through the bronchoscope. After the instillation of each aliquot, instilled saline will be retrieved with a negative suction pressure of less than $100 \mathrm{mmHg}$ that can avoid visible airway collapse. The retrieved BALF (at least $5 \mathrm{~mL}$ ) will be subdivided into aliquots within $1 \mathrm{~h}$ of collection after being shaken to ensure homogeneous mixing, and stored at $-80{ }^{\circ} \mathrm{C}$ for GM detection.

\section{GM detection}

Peripheral venous blood will be collected into Vacutainer ${ }^{\curvearrowleft}$ tubes (BD Worldwide, Suzhou, China), and serum will be obtained by centrifugation at $3000 \mathrm{rpm}, 4{ }^{\circ} \mathrm{C}$ for 10 mins. The GM assay on serum, BALF and sputum will be performed with the Platelia Aspergillus enzyme immunoassay (Bio-Rad, California, USA) according to the manufacturer's instructions. Tests for serum and BALF GM will be considered positive at cut-off values of $\geq 0.5$ ODI and $\geq 1.0$ ODI, respectively.

\section{LFD performance}

LFD will be performed as Thornton et al. described [36]. Briefly, $100 \mu \mathrm{L}$ pretreated serum (1:1 diluted with tissue culture medium), neat BALF or processed sputum supernatant will be added to the release port on the LFD device after gentle mixing and will be incubated at room temperature for 15 mins. The development of the control line in the result window shows that the test has run correctly, and the development of the Aspergillus-specific test line will be determined after 15 mins. The results will be recorded as positive if the test line is present, or be recorded as negative if the test line is absent. Each LFD result will be assessed by two researchers independently. 


\section{A-DNA extraction and RT-PCR}

Fungal DNA will be extracted from $1.5 \mathrm{~mL}$ of the sputum suspension with the commercial MycXtra fungal DNA extraction kit (Myconostica Limited, Manchester, UK), and $A$-DNA will be detected with the MycAssay Aspergillus kit (Myconostica Limited, Manchester, UK), according to the manufacturer's instructions. PCR runs will be performed on a CFX Connect ${ }^{\mathrm{TM}}$ RT-PCR system (Bio-Rad, California, USA) by targeting a portion of the $18 \mathrm{~S}$ ribosomal gene, in accordance with the detection kit protocol. Ten microliters of extracted DNA will be used in a final reaction volume of $25 \mu \mathrm{L}$. The PCR protocol is as follows: 10 mins at $94{ }^{\circ} \mathrm{C} ; 10 \mathrm{~s}$ at $94{ }^{\circ} \mathrm{C}, 58 \mathrm{~s}$ at $57{ }^{\circ} \mathrm{C}$, and $20 \mathrm{~s}$ at $72{ }^{\circ} \mathrm{C}$ for 40 cycles. The cycling threshold will be recorded and used for subsequent analysis, including the determination of an optimal cut-off value, based on which the sputum samples will be regarded as positive or negative for Aspergillus infection.

\section{TAFC and bmGT detection}

High-performance liquid chromatography tandem mass spectrometry (HPLC-MS/MS) on Agilent 1260-6460 (Agilent Technologies Inc., Santa Clara, USA) was first developed in our laboratory and will be used to simultaneously analyse TAFC and bmGT from sputum. The method employs phenacetin as the internal standard, and shows the lower and upper limits of quantitation for TAFC and bmGT assays of $1.56 \mathrm{ng} / \mathrm{mL}$ and $100 \mathrm{ng} / \mathrm{mL}$, respectively. Upon detection, $100 \mu \mathrm{L}$ of sputum will be spiked with phenacetin, vortexed and extracted with $400 \mu \mathrm{L}$ of dichloromethane. The extraction process will be repeated once, and the total solvent will be evaporated with a pressure gas blowing concentrator. The residue will then be reconstituted in $100 \mu \mathrm{L}$ of acetonitrile/water $(50 / 50 \mathrm{v} / \mathrm{v} \%)$, vortexed and centrifuged. Three microliters of supernatant will be injected for HPLC-MS/MS analysis. Standards of TAFC (EMC, Germany) and bmGT (Enzo Life Sciences, Inc., USA) are spiked into sputum samples from four patients with bacterial pneumonia to evaluate the recovery of both compounds. Each of the four sputum samples is divided into three aliquots, with one aliquot as a control and the other two spiked with different concentrations of TAFC and bmGT. We assayed one sputum sample from a patient diagnosed with lung cancer (squamous cell carcinoma) and probable IPA using the HPLC-MS/MS method.

\section{Clinical follow-up}

Patients will receive telephone reviews at 3 months and 6 months after enrolment. Information that includes the patients' symptoms, days of hospitalization and survival will be recorded and checked in the medical record system.

\section{Sample size calculation}

GM is the most studied biomarker for the diagnosis of IPA among our candidate signatures; therefore, the sample size calculation is based on the diagnostic accuracy of the sputum GM assay reported by previous studies. Receiver operating characteristics (ROC) curves show the trade-off between sensitivity and specificity, and the area under the curve (AUC) is considered as an overall index of accuracy for quantitative biomarkers. The sample size estimation is based on AUC reported previously [27].

With $95 \%$ confidence $(\alpha=0.05)$ to guarantee the marginal error of estimate (i.e. the difference between true AUC and its estimate) does not exceed from a pre-determined value of $d=0.09$, and expecting an equal number of diseased and non-diseased cases, the required sample size for each group is:

$$
\mathrm{n}=\frac{\mathrm{z}_{\frac{\alpha}{2}}^{2} \mathrm{~V}(\widehat{\mathrm{AUC}})}{d^{2}}
$$

$\mathrm{V}(\widehat{\mathrm{AUC}})$ denotes the variance of $\widehat{\mathrm{AUC}}$ that is estimated parametrically based on binormal assumption [41, 42]. This binormal assumption is chosen because our study implements a four-category rating scheme (i.e. proven IPA, probable IPA, possible IPA and no IPA $). V(\widehat{A U C})$ is calculated as follows:

$$
\begin{aligned}
\mathrm{V}(\widehat{\mathrm{AUC}})= & \left(0.0099 \times e^{-a^{2} / 2}\right) \\
& \times\left(6 a^{2}+16\right)
\end{aligned}
$$

where $\mathrm{a}=\phi^{-1}(\mathrm{AUC}) \times 1.414$ and $\phi^{-1}$ is the inverse of standard cumulative normal distribution. With a pre-specified AUC of 0.795 according to Kimura et al. [27], the required sample size for each group is 59 after above calculation. Therefore, we will recruit a minimum number of 118 participants with 59 controls (no IPA) and 59 cases (proven or probable IPA).

\section{Statistical analysis}

The data will be analysed with SPSS 22.0 software (IBM Corp, Armonk, NY, USA). Two analyses will be performed based on the IPA classification, one in which patients with possible IPA will be excluded in the calculation and a second analysis in which patients with possible IPA will be classified as not having IPA. Therefore, the comparisons and analyses will be (1) proven/ probable IPA versus no IPA, and (2) proven/probable IPA versus possible/no IPA [43]. The sensitivity, specificity, negative predictive value (NPV), positive predictive value (PPV), and diagnostic odds ratio, including 95\% confidence intervals, will be calculated for different biomarkers. ROC analysis will be performed and AUC 
(including 95\% CI) and cut-off values will be established from the ROC curves for GM, RT-PCR, TAFC and bmGT. The McNemar $\chi^{2}$ test will be used to compare sensitivity, specificity, NPV, and PPV between different assays. The difference in hospital stay and the survival between possible or no IPA versus proven or probable IPA will be analysed with chi-square tests, and the relationships between the levels of biomarkers and the hospital stay and survival will be analysed via regression models. $P<0.05$ will be defined as statistically significant.

\section{Results of pilot study}

Sputa used for spiking tests were obtained from four patients who were not diagnosed with IPA. TAFC and bmGT were undetectable in these samples. The detected concentrations of the $5 \mathrm{ng} / \mathrm{mL}$ and $40 \mathrm{ng} / \mathrm{mL}$ TAFC and bmGT standards spiked into the sputa from the four no IPA patients are presented in Table 4 . The method was found to be accurate and precise and has high recovery for both TAFC and bmGT from spiked sputum samples (Table 4). The accuracy (\%deviation), precision (\%RSD) and recovery (\%) with our protocol for TAFC/bmGT detection at a $5 \mathrm{ng} / \mathrm{mL}$ level were $\leq \pm 5.0 / 5.0 \%, 5.2 / 7.3 \%$ and $98.4 / 96.9 \%$, respectively.

Sputum culture for the patient with lung cancer and probable IPA yielded Aspergillus fumigatus twice while the serum GM showed negative result $(\mathrm{ODI}=0.12)$. TAFC and bmGT were detectable in this patient's sputum and the concentrations are $45.36 \mathrm{ng} / \mathrm{mL}$ and $46.35 \mathrm{ng} / \mathrm{mL}$, respectively (Fig. 3).

\section{Discussion}

The clinical and radiological features of IPA are atypical, leading to the difficulty in an early diagnosis and treatment of the disease. There are still uncertainties and controversies associated with the diagnosis of IPA in clinical practice. Intensive efforts have been made to improve the diagnosis in "high-risk" individuals such as haematological and immunocompromised patients. Consequently, a series of approaches have been developed attempting to access a precise diagnosis.

Biomarker development has opened new possibilities in the diagnosis of a great number of disorders including IPA. Biomarkers related to IPA have been isolated and investigated largely in serum or BALF sample, yet few study has addressed the possibility of sputum biomarkers in the diagnosis of IPA. Each sample type has its advantages as well as disadvantages (Table 5). Biomarker detection using serum is convenient, inexpensive and time-saving, presenting high specificity [20], however the sensitivity was reported to be low in non-haematological patients $[6,23,44,45]$. In comparison, BALF assays exhibit higher sensitivity as well as specificity in both neutropenic [46] and non-neutropenic patients $[7,9]$, probably because BALF is retrieved from local airways. However, the bronchoscopy is an invasive and costly procedure, and it might not be accessible to severe patients or those with contraindications.

Sputum is the phlegm from the respiratory tract that has altered macromolecular, polymer composition and biophysical properties varying with diseases. Studies investigating sputum biomarkers are often limited by the techniques to obtain the specimen; however, intensive studies have demonstrated that hypertonic saline-induced sputum can be processed to yield reproducible results that reflect disease activity and correlate with BALF [47]. Aspergillus-related biomarkers, including $A$-DNA and GM, have been investigated using sputum samples, and we have developed a method to detect TAFC and bmGT in sputum; therefore, it is technically possible to use sputum for biomarker detection in the diagnosis of IPA.

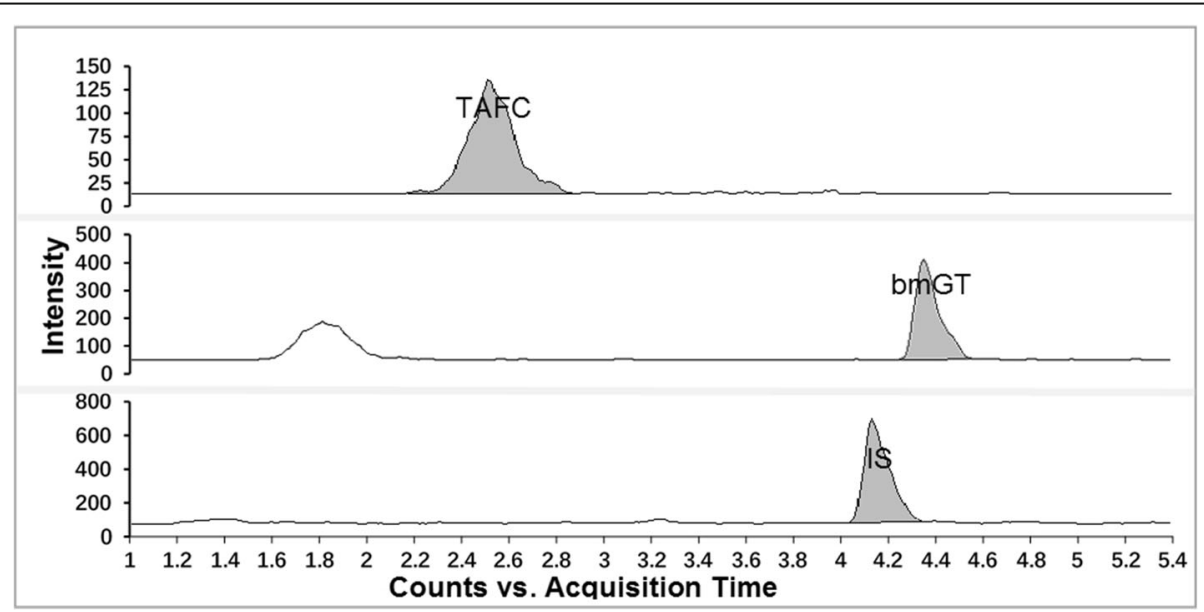

Fig. 3 HPLC-MS/MS detection of TAFC and bmGT in the sputum of a probable IPA patient. Abbreviations: bmGT=bis(methylthio)gliotoxin; TAFC = triacetylfusarinine; $I S=$ internal standard (phenacetin) 
Table 4 Evaluation of accuracy, precision, and recovery of TAFC and bmGT from spiked sputum samples (mean \pm SD)

\begin{tabular}{lllll}
\hline $\begin{array}{l}\text { Spiked TAFC \& bmGT } \\
\text { (ng/mL) }\end{array}$ & $\begin{array}{l}\text { Detected TAFC } \\
\text { (\%deviation) }\end{array}$ & $\begin{array}{l}\text { Detected bmGT } \\
\text { (\%deviation) }\end{array}$ & $\begin{array}{l}\text { TAFC Recovery } \\
\%(\% R S D)\end{array}$ & $\begin{array}{l}\text { bmGT Recovery } \\
\%(\% R S D)\end{array}$ \\
\hline 0 & Not detected & Not detected & Not applicable & Not applicable \\
5 & $4.9 \pm 0.3(-1.6)$ & $4.8 \pm 0.4(-3.1)$ & $98.4 \pm 0.1(5.2)$ & $96.9 \pm 0.1(7.3)$ \\
40 & $38.7 \pm 1.2(-3.3)$ & $39.0 \pm 2.9(-2.5)$ & $96.7 \pm 3.0(3.1)$ & $97.5 \pm 7.3(7.4)$ \\
\hline
\end{tabular}

Although no studies have investigated the superiority of the sputum assay over serum or BALF for the diagnosis of IPA in non-haematological patients, the sputum assay might be a valuable and useful approach for the detection of Aspergillus infection in patients with pre-existing respiratory diseases due to the following reasons. First, non-neutropenic patients tend to develop airway invasive forms of IPA [24, 25], which implies that airway-retrieved samples might be more sensitive to Aspergillus constituents. Additionally, Escribano et al. [48] identified matching genotypes of Aspergillus fumigatus in BALF and the sputum/bronchial secretion samples in proven or probable IPA patients, which indicates a comparable accuracy of BALF and sputum detection for the diagnosis of IPA. Furthermore, in the study by Kimura et al. [27], sputum GM was elevated in all patients with proven or probable IPA, and sputum GM showed a higher concentration (average GM index of 4.5) than that in BALF and serum (1.4 and 1.0, respectively). The above evidence strongly supports the usefulness of the sputum assay for the diagnosis of IPA.

Potential diagnostic biomarkers for IPA have been intensively studied, given the problem of an early diagnosis. We have reviewed the literature systematically to identify potential biomarkers that can be detected in a sputum sample. Galactomannan (GM) is a cell wall polysaccharide that is released by Aspergillus during fungal growth. The method of GM detection has been commercialized and widely used in the clinic with serum and BALF samples [19]. The serum GM assay exhibits low sensitivity (23.1-42\%) for the diagnosis of IPA in non-haematological patients [6, 23], whereas BALF GM shows higher sensitivity and specificity [6-9]. The GM assay for sputum samples has been established in several studies (Table 1). Whether a GM assay based on lower airway derived sputum can be used as a non-invasive tool for the diagnosis of IPA deserves investigation. The measurement of GM in sputum might provide a more sensitive and non-invasive diagnostic approach for IPA.

GM detection can be affected by non-Aspergillus fungi and food that contains GM and beta-lactam antibiotics, which can produce false-positive values [49-51]. Several other biomarkers have been shown to play a role in the diagnosis of IPA and may be potential candidates for future clinical use. A rapid detection with LFD is an immunochromatographic assay that uses a monoclonal antibody called JF5 that targets an extracellular glycoprotein secreted during active fungal growth [36]. The test can be performed bedside and accomplished within 15 mins and, therefore, can be used as an early indicator for Aspergillus infection. The device is highly specific to antigens from Aspergillus but does not react with antigens from a large number of clinically important fungi, especially Candida species [36]. Pan et al. [52] conducted a meta-analysis to investigate its diagnostic accuracy for invasive aspergillosis, and showed that the LFD assay using BALF had higher sensitivity (86\%) and specificity (93\%) than serum (68\% sensitivity and $87 \%$ specificity). Importantly, the BALF LFD assay also exhibited satisfying diagnostic performance for IPA (77\% sensitivity and 92\% specificity) in patients with URD [11]. Sputum supernatant has a similar matrix as BALF after processing procedures; hence, the use of the sputum LFD assay may provide a bedside and time-saving diagnostic tool for IPA.

TAFC is a siderophore secreted soon after conidiospore germination by Aspergillus in iron-limited media and is essential for fungal virulence [53, 54]. In a rat model of Aspergillus fumigatus infection, (68)Ga radiolabelled Aspergillus TAFC selectively accumulates in infected lung tissue [55]. Recently, Carrol et al. [37] detected TAFC in the serum of patients at risk for

Table 5 Pros and cons of serum, BALF and sputum samples for IPA diagnosis in non-haematological patients

\begin{tabular}{|c|c|c|}
\hline Samples & Pros & Cons \\
\hline Serum & $\begin{array}{l}\square \text { Convenient, cheap and fast } \\
\square \text { High specificity }\end{array}$ & aLow sensitivity \\
\hline BALF & $\begin{array}{l}\text { 口Local-infection related } \\
\square \text { High sensitivity and specificity }\end{array}$ & $\begin{array}{l}\text { 口Invasive, having contraindications } \\
\square \text { Expensive }\end{array}$ \\
\hline Sputum & $\begin{array}{l}\square \text { Convenient, cheap and fast } \\
\square \text { Local-infection related } \\
\square \text { Non-invasive }\end{array}$ & $\square$ Affected by colonization and contamination \\
\hline
\end{tabular}


invasive aspergillosis using ultra-performance liquid chromatography tandem mass spectrometry. TAFC might be a potential early diagnostic biomarker for IPA. Gliotoxin (GT) is the major and the most potent toxin produced by Aspergillus fumigatus and mediates immunosuppressive and proapoptotic effects that may promote the establishment of invasive aspergillosis [56-58]; however, the rapid elimination of GT from body fluids by adjoining tissues and circulating cells precludes its feasibility as a biomarker for IPA [59-61]. bmGT is the inactive metabolite derived from GT and is quite stable after secreted, therefore it has been studied for the possibility of being an alternative biomarker [61]. Matxalen et al. [62] found that serum bmGT presented higher sensitivity (61.5\%) and similar specificity (93\%) compared to serum GM in haematological patients for the diagnosis of IPA. The assays for TAFC and bmGT have not yet been developed and validated in sputum. After a series of strict experiments, we first identified and standardized the TAFC and bmGT assays in sputum via the HPLC-MS/MS technique. We further validated the methods by determining the sputa induced from a group of patients with probable IPA or no IPA, which is consistent with the clinical diagnosis showing the great potential of being a non-invasive and useful marker for IPA. The detailed methods to process sputum and detect TAFC and bmGT in sputum have been submitted for patents (State Intellectual Property Office in China, No.201710428772.9). In this study, the diagnostic performance of sputum TAFC and bmGT assays for the diagnosis of IPA in patients with underlying respiratory disease will first be examined.

RT-PCR is a precise approach to detect $A$-DNA, and it has been applied for serum and BALF samples even though there is a lack of standardized protocol. Several meta-analyses [21, 63-67] assessed the efficacy of PCR assays on these two sample types for the diagnosis of IPA in haematological patients. The results showed that BALF PCR had much higher sensitivity (approximately $90 \%)$ and specificity (>90\%) than serum (85\% for sensitivity and $75 \%$ for specificity). Several studies [28, 29, 68, 69] used PCR to identify Aspergillus in sputum and showed that sputum PCR had higher sensitivity than routine fungal culture; however, this technique has not been applied in the diagnosis of IPA and will be addressed in our study.

Sputum is a type of sample that is viscous and heterogeneous. To accurately detect biomarkers in sputum plugs, a proper processing method is needed. Dithiothreitol (DDT) is a commonly used reagent to homogenize sputum before all kinds of constituents are examined, and it is recommended for the pre-treatment of viscous samples by guidelines [70]. Baxter et al. [28] processed sputum with DDT and sonication before performing a
GM assay and the results showed excellent GM reproducibility and low interassay and intraassay coefficients of variation. However, Prattes et al. [71] examined the effect of DDT on a BALF GM assay and found conflicting results. GM levels in BALF were substantially decreased from 0.51 ODI to 0.01 ODI after pre-treatment with DTT, and the LFD assay in BALF was also dramatically affected. Although this study targeted BALF, it is likely that DTT might affect sputum GM and LFD detection. Therefore, to minimize the effect of DTT on these biomarker assays, we will pre-treat sputum samples with phosphate buffered saline, which is in accordance with the method by Kimura et al. [27].

There are concerns about the specificity of the sputum assay related to colonization or contamination. Nevertheless, the four biomarkers (GM, JF5 antigen, TAFC, bmGT) in our study are produced and released during fungal growth. It is less likely that the increase in the levels of these biomarkers is a result of contamination of fungal spores. In addition, lower airway secretions will be used by sputum induction, and sputum quality control by squamous cell counting and viability calculation will further exclude the possibility of contamination. We will also determine an appropriate cut-off value of different sputum biomarkers, which will help to differentiate colonization from infection.

There are several highlights of this study. We systematically evaluate the validity of various biomarkers based on sputum from lower airways for the diagnosis of IPA for the first time; in particular, the establishment of a method that simultaneously detects TAFC and bmGT in sputum is first described and applied for patents and reported. Furthermore, the study population, i.e., patients with URD, has been increasingly recognized to present with IPA, which is associated with high mortality [12], but only has been addressed in a small number of studies. We assess the sputum biomarkers for IPA diagnosis in this population for the first time, and the results may help to provide novel, non-invasive and valuable diagnostic tools for IPA in patients with URD. In addition to sputum, serum and BALF will also be collected and compared for their diagnostic performance. In the statistical analysis, we will conduct two different analyses to investigate the impact of the possible IPA group on the interpretation of the results. Except for the investigation of diagnostic efficacy, we will also conduct a clinical follow-up to observe the prognosis of different types of IPA and further investigate host factors predisposing to IPA in patients with URD.

This study has some limitations that should be considered. The categories of probable and possible IPA are proposed for immunocompromised patients only in the consensus report by the EORTC/MSG. However, this report recognizes that the revised definition of IPA does 
not necessarily apply to immunocompetent patients as it is difficult to find a sufficient basis for identifying appropriate host factors. Patients with URD may possess much different risk factors to Aspergillus infection compared to those immunocompromised individuals. As in the study by Prattes et al. [11], pre-existing respiratory disease is added as an independent host factor to establish the diagnosis in our study. We will also conduct post hoc analyses of the host factors linked to diagnosis and prognosis for further investigation. The concentration of biomarkers in the sputum may fluctuate with the production and expectoration of the sputum and the releasing of the biomarkers from the infection site. However, this study already includes a great number of biomarkers from different sample types with a comparable large sample size, and we do not perform serial tests for sputum biomarkers at different time points across the disease course, which may lead to the underestimation of the diagnostic performance of several biomarkers. Further study may be warranted to investigate the dynamic variation of different Aspergillus biomarkers in sputum.

In conclusion, the design and protocol of a clinical trial that investigates the performance of sputum signatures for the diagnosis and prognosis of IPA in patients with URD are presented here. The results of this innovative study are expected to provide answers to the following questions. Are sputum signatures valuable diagnostic tools for IPA in patients with URD? Which signature is the strongest potential candidate? Would the modified host factors be appropriate for this patient population? What disease course of IPA would present in this population? The study findings will provide evidence and novel insights into these areas, which have not been accessed.

\section{Abbreviations \\ A-DNA: Aspergillus DNA; AUC: Area under the curve; BALF: Bronchoalveolar lavage fluid; bmGT: Bis(methylthio)gliotoxin; COPD: Chronic obstructive pulmonary disease; DDT: Dithiothreitol; EORTC/MSG: European Organization for Research and Treatment of Cancer/Mycoses Study Group; GM: Galactomannan; HPLC-MS/MS: High-performance liquid chromatography tandem mass spectrometry; IPA: Invasive pulmonary aspergillosis; LFD: Lateral flow device; NPV: Negative predictive value; ODI: Optical density index; PCR: Polymerase chain reaction; PPV: Positive predictive value; ROC: Receiver operating characteristics; RT-PCR: Real time polymerase chain reaction; SPARED: Sputum signatures for invasive pulmonary aspergillosis in patients with underlying respiratory diseases; TAFC: Triacetylfusarinine; URD: Underlying respiratory diseases}

\section{Acknowledgements}

The authors acknowledge Dr. Christopher R. Thornton (University of Exeter, UK) for providing testing LFD device and technique consultation of the assay using LFD for our study; and Dao-hong Chen for the work on clinical recruitment and sample processing.

\section{Trial status}

The trial was registered in August 2016, and is currently waiting for participant recruitment. The results of this study will be available in 2020 .

\section{Funding}

This trial is financially supported by the Thousand Talents Program, Sichuan province, China (0082204151045). The funder has no role in the design of the study and writing the manuscript and will not involve in the collection, analysis, and interpretation of data, and in any decision to publish the results.

Availability of data and materials

All data generated or analysed during this study are included in this published article.

\section{Authors' contributions}

JJF participated in the study design and drafted the manuscript. WX contributed to the study design, trial registration, ethics application and manuscript writing. DYG contributed to the development of the method for TAFC and bmGT assays using HPLC-MS/MS technique, and the sample determination. BM and XMD participated in the study design and the supervision of clinical recruitment. LLC and MYW participated in the protocol development, participant recruitment and sample processing. All authors read and approved the final manuscript.

\section{Ethics approval and consent to participate}

This trial will be conducted in accordance with the Declaration of Helsinki. The Clinical Trial and Biomedical Ethics Committee of West China Hospital of Sichuan University approved the study (No 2016-234). All participants will be volunteers, and a written informed consent form will be signed. Participants will be able to cease their involvement in the study at any time. If there are any changes to the protocol during the course of the study, an amendment will be submitted to the institutional review board for approval.

\section{Competing interests}

The authors declare that they have no competing interests.

\section{Publisher's Note}

Springer Nature remains neutral with regard to jurisdictional claims in published maps and institutional affiliations.

\section{Author details}

${ }^{1}$ Respiratory Group, Department of Integrated Traditional Chinese and Western Medicine, West China Hospital, Sichuan University, Chengdu, China. ${ }^{2}$ Laboratory of Anesthesia and Critical Care Medicine, Translational Neuroscience Center, West China Hospital, Sichuan University, Chengdu, China. ${ }^{3}$ Department of Respiratory and Critical Care Medicine, West China Hospital, Sichuan University, Chengdu, China.

Received: 30 June 2017 Accepted: 30 May 2018

Published online: 11 June 2018

\section{References}

1. Kontoyiannis DP, Marr KA, Park BJ, Alexander BD, Anaissie EJ, Walsh TJ, et al. Prospective surveillance for invasive fungal infections in hematopoietic stem cell transplant recipients, 2001-2006: overview of the transplant-associated infection surveillance network (TRANSNET) database. Clin Infect Dis. 2010; 50(8):1091-100.

2. Meersseman W, Vandecasteele SJ, Wilmer A, Verbeken E, Peetermans WE, Van Wijngaerden E. Invasive aspergillosis in critically ill patients without malignancy. Am J Respir Crit Care Med. 2004;170(6):621-5.

3. Taccone FS, Van den Abeele AM, Bulpa P, Misset B, Meersseman W, Cardoso $\mathrm{T}$, et al. Epidemiology of invasive aspergillosis in critically ill patients: clinical presentation, underlying conditions, and outcomes. Crit Care. 2015;19:7.

4. Delsuc C, Cottereau A, Frealle E, Bienvenu AL, Dessein R, Jarraud S, et al. Putative invasive pulmonary aspergillosis in critically ill patients with chronic obstructive pulmonary disease: a matched cohort study. Crit Care. 2015;19:421.

5. Tejeda MI, Salso S, Barberan J. Invasive pulmonary aspergillosis in nonneutropenic patients. Revista Espanola de Quimioterapia. 2016;29(1):56-8.

6. Meersseman W, Lagrou K, Maertens J, Wilmer A, Hermans G, Vanderschueren S, et al. Galactomannan in bronchoalveolar lavage fluid: a tool for diagnosing aspergillosis in intensive care unit patients. Am J Respir Crit Care Med. 2008;177(1):27-34. 
7. Ozger S, Hizel K, Kalkanci A, Aydogdu M, Civil F, Dizbay M, et al. Evaluation of risk factors for invasive pulmonary aspergillosis and detection of diagnostic values of galactomannan and PCR methods in bronchoalveolar lavage samples from non-neutropenic intensive care unit patients. Mikrobiyoloji Bulteni. 2015;49(4):565-75.

8. Zhang S, Wang S, Wan Z, Que C, Li R, Yu J. Quantitative real-time PCR and Platelia galactomannan assay for the diagnosis of invasive pulmonary aspergillosis: bronchoalveolar lavage fluid performs better than serum in non-neutropaenic patients. Mycopathologia. 2016;181(9-10):625-9.

9. Fortun J, Martin-Davila P. Gomez Garcia de la Pedrosa E, Silva JT, GarciaRodriguez J, Benito D, et al. galactomannan in bronchoalveolar lavage fluid for diagnosis of invasive aspergillosis in non-hematological patients. J Infection. 2016:72(6):738-44.

10. Guinea J, Torres-Narbona M, Gijon P, Munoz P, Pozo F, Pelaez T, et al. Pulmonary aspergillosis in patients with chronic obstructive pulmonary disease: incidence, risk factors, and outcome. Clin Microbiol Infect. 2010; 16(7):870-7.

11. Prattes J, Flick H, Pruller F, Koidl C, Raggam RB, Palfner M, et al. Novel tests for diagnosis of invasive aspergillosis in patients with underlying respiratory diseases. Am J Respir Crit Care Med. 2014;190(8):922-9.

12. Cornillet A, Camus C, Nimubona S, Gandemer V, Tattevin P, Belleguic C, et al. Comparison of epidemiological, clinical, and biological features of invasive aspergillosis in neutropenic and nonneutropenic patients: a 6-year survey. Clin Infect Dis. 2006;43(5):577-84.

13. Samarakoon P, Soubani A. Invasive pulmonary aspergillosis in patients with COPD: a report of five cases and systematic review of the literature. Chron Respir Dis. 2008;5(1):19-27.

14. von Eiff M, Roos N, Schulten R, Hesse M, Zuhlsdorf M, van de Loo J. Pulmonary aspergillosis: early diagnosis improves survival. Respiration. 1995; 62(6):341-7.

15. Girmenia C, Guerrisi P, Frustaci AM, Fama A, Finolezzi E, Perrone S, et al. New category of probable invasive pulmonary aspergillosis in haematological patients. Clin Microbiol Infect. 2012;18(10):990-6.

16. Ruhnke M, Bohme A, Buchheidt D, Donhuijsen K, Einsele H, Enzensberger $R$, et al. Diagnosis of invasive fungal infections in hematology and oncology-guidelines of the infectious diseases working party $(\mathrm{AGIHO})$ of the German Society of Hematology and Oncology (DGHO). Ann Hematol. 2003;82(2):S141-8.

17. De Pauw B, Walsh TJ, Donnelly JP, Stevens DA, Edwards JE, Calandra T, et al. Revised definitions of invasive fungal disease from the European Organization for Research and Treatment of Cancer/invasive fungal infections cooperative group and the National Institute of Allergy and Infectious Diseases mycoses study group (EORTC/MSG) consensus group. Clin Infect Dis. 2008;46(12):1813-21.

18. Tarrand JJ, Lichterfeld M, Warraich I, Luna M, Han XY, May GS, et al. Diagnosis of invasive septate mold infections. A correlation of microbiological culture and histologic or cytologic examination. Am J Clin Pathol. 2003;119(6):854-8.

19. Mennink-Kersten MA, Donnelly JP, Verweij PE. Detection of circulating galactomannan for the diagnosis and management of invasive aspergillosis. Lancet Infect Dis. 2004:4(6):349-57.

20. Leeflang MM, Debets-Ossenkopp YJ, Wang J, Visser CE, Scholten RJ, Hooft L, et al. Galactomannan detection for invasive aspergillosis in immunocompromised patients. Cochrane database Syst Rev. 2015; https:// doi.org/10.1002/14651858.CD007394.pub2.

21. Mengoli C, Cruciani M, Barnes RA, Loeffler J, Donnelly JP. Use of PCR for diagnosis of invasive aspergillosis: systematic review and meta-analysis. Lancet Infect Dis. 2009;9:89-96.

22. Imbert S, Gauthier L, Joly I, Brossas JY, Uzunov M, Touafek F, Brun S, et al. Aspergillus PCR in serum for the diagnosis, follow-up and prognosis of invasive aspergillosis in neutropenic and nonneutropenic patients. Clin Microbiol Infect. 2016;22(6):562

23. Ku NS, Han SH, Choi JY, Kim SB, Kim HW, Jeong SJ, et al. Diagnostic value of the serum galactomannan assay for invasive aspergillosis: it is less useful in non-haematological patients. Scand J Infect Dis. 2012;44(8):600-4.

24. Bergeron A, Porcher R, Sulahian A, de Bazelaire C, Chagnon K, Raffoux E, et al. The strategy for the diagnosis of invasive pulmonary aspergillosis should depend on both the underlying condition and the leukocyte count of patients with hematologic malignancies. Blood. 2012;119(8):1831-7.

25. Petraitiene R, Petraitis V, Bacher JD, Finkelman MA, Walsh TJ. Effects of host response and antifungal therapy on serum and BAL levels of galactomannan and (1,3)-beta-D-glucan in experimental invasive pulmonary aspergillosis. Med Mycol. 2015;53(6):558-68.

26. He H, Ding L, Sun B, Li F, Zhan Q. Role of galactomannan determinations in bronchoalveolar lavage fluid samples from critically ill patients with chronic obstructive pulmonary disease for the diagnosis of invasive pulmonary aspergillosis: a prospective study. Crit Care. 2012;16(4):R138.

27. Kimura S, Odawara J, Aoki T, Yamakura M, Takeuchi M, Matsue K. Detection of sputum aspergillus galactomannan for diagnosis of invasive pulmonary aspergillosis in haematological patients. Int J Hematol. 2009;90(4):463-70.

28. Baxter CG, Dunn G, Jones AM, Webb K, Gore R, Richardson MD, et al. Novel immunologic classification of aspergillosis in adult cystic fibrosis. J Allergy Clin Immunol. 2013;132(3):560-6.

29. Baxter CG, Rautemaa R, Jones AM, Webb AK, Bull M, Mahenthiralingam E, et al. Intravenous antibiotics reduce the presence of aspergillus in adult cystic fibrosis sputum. Thorax. 2013:68(7):652-7.

30. Farrant JP, Wardman $H$, Holmes L, Chishimba L, Niven R. Aspergillus polymerase chain reaction testing in the severe asthma population. Thorax. 2012;67:A31

31. Farrant J, Brice H, Fowler S, Niven R. Fungal sensitisation in severe asthma is associated with the identification of aspergillus fumigatus in sputum. J Asthma. 2016:53(7):732-5.

32. Fraile Farinas MT, Chanza Avino M, Ramos Marti JL, Celma Arminana I, Ocete Mochon MD, Gomez Munoz N, et al. Invasive aspergillosis: evaluation of several diagnosis tests. Clinical microbiology and infection conference: 22nd European congress of clinical microbiology and. Infectious Dis. 2012; 18:139-40.

33. Chanza M, Fraile MT, Gimeno C, Ocete MD. Evaluation of galactomannan antigen and aspergillus real time PCR for diagnosis of invasive aspergillosis. Revista Espanola de Quimioterapia. 2014;27(1):43-5.

34. Delfino E, Favilli F, Cresta F, Casciaro R, Furfaro E, Minicucci L, et al. Sputum galactomannan in cystic fibrosis patients colonized with aspergillus fumigatus. Pediatr Pulmonol. 2012:47:341.

35. Dhillon R, Speight L, Duckers J, White PL. An evaluation of the performance of a galactomannan (GM) assay in aiding the diagnosis of allergic bronchopulmonary aspergillosis (ABPA) in cystic fibrosis (CF) patients. J Cyst Fibros. 2016;15:S78-9.

36. Thornton CR. Development of an immunochromatographic lateral-flow device for rapid serodiagnosis of invasive aspergillosis. Clin Vaccine Immunol. 2008;15(7):1095-105.

37. Carroll CS, Amankwa LN, Pinto LJ, Fuller JD, Moore MM. Detection of a serum Siderophore by LC-MS/MS as a potential biomarker of invasive aspergillosis. PLoS One. 2016;11(3):e0151260.

38. Gibson PG, Wlodarczyk JW, Hensley MJ, Gleeson M, Henry RL, Cripps AW, et al. Epidemiological association of airway inflammation with asthma symptoms and airway hyperresponsiveness in childhood. Am J Respir Crit Care Med. 1998;158(1):36-41.

39. Pashley CH, Fairs A, Morley JP, Tailor S, Agbetile J, Bafadhel M, et al. Routine processing procedures for isolating filamentous fungi from respiratory sputum samples may underestimate fungal prevalence. Med Mycol. 2012; 50(4):433-8

40. Meyer KC, Raghu G, Baughman RP, Brown KK, Costabel U, du Bois RM, et al. An official American Thoracic Society clinical practice guideline: the clinical utility of bronchoalveolar lavage cellular analysis in interstitial lung disease. Am J Respir Crit Care Med. 2012;185(9):1004-14

41. Hajian-Tilaki K. Sample size estimation in diagnostic test studies of biomedical informatics. J Biomed Inform. 2014;48:193-204.

42. Obuchowski NA. Computing sample size for receiver operating characteristic studies. Investig Radiol. 1994;29(2):238-43.

43. Lehrnbecher T, Robinson PD, Fisher BT, Castagnola E, Groll AH, Steinbach WJ, et al. Galactomannan, beta-D-glucan, and polymerase chain reactionbased assays for the diagnosis of invasive fungal disease in pediatric cancer and hematopoietic stem cell transplantation: a systematic review and metaanalysis. Clin Infect Dis. 2016;63(10):1340-8.

44. Zhou W, Li H, Zhang Y, Huang M, He Q, Li P, et al. Diagnostic value of the galactomannan antigen test in serum and bronchoalveolar lavage fluid samples from patients with non-neutropenic invasive pulmonary aspergillosis. J Clin Microbiol. 2017; https://doi.org/10.1128/ jcm.00345-17.

45. Prattes J, Hoenigl M, Krause R, Buzina W, Valentin T, Reischies F, et al. Invasive aspergillosis in patients with underlying liver cirrhosis: a prospective cohort study. Med Mycol. 2017; https://doi.org/10.1093/mmy/myx011. 
46. Boch T, Buchheidt D, Spiess B, Miethke T, Hofmann WK, Reinwald M. Direct comparison of galactomannan performance in concurrent serum and bronchoalveolar lavage samples in immunocompromised patients at risk for invasive pulmonary aspergillosis. Mycoses. 2016;59(2):80-5.

47. Fahy JV, Wong H, Liu J, Boushey HA. Comparison of samples collected by sputum induction and bronchoscopy from asthmatic and healthy subjects. Am J Respir Crit Care Med. 1995;152(1):53-8.

48. Escribano P, Marcos-Zambrano LJ, Pelaez T, Munoz P, Padilla B, Bouza E, et al. Sputum and bronchial secretion samples are equally useful as bronchoalveolar lavage samples for the diagnosis of invasive pulmonary aspergillosis in selected patients. Med Mycol. 2015;53(3):235-40.

49. Giacchino M, Chiapello N, Bezzio S, Fagioli F, Saracco P, Alfarano A, et al. Aspergillus galactomannan enzyme-linked immunosorbent assay cross-reactivity caused by invasive Geotrichum capitatum. J Clin Microbiol. 2006;44(9):3432-4.

50. Aubry A, Porcher R, Bottero J, Touratier S, Leblanc T, Brethon B, et al. Occurrence and kinetics of false-positive aspergillus galactomannan test results following treatment with beta-lactam antibiotics in patients with hematological disorders. J Clin Microbiol. 2006;44(2):389-94.

51. Mattei D, Rapezzi D, Mordini N, Cuda F, Lo Nigro C, Musso M, et al. Falsepositive aspergillus galactomannan enzyme-linked immunosorbent assay results in vivo during amoxicillin-clavulanic acid treatment. J Clin Microbiol. 2004;42(11):5362-3.

52. Pan Z, Fu M, Zhang J, Zhou H, Fu Y, Zhou J. Diagnostic accuracy of a novel lateral-flow device in invasive aspergillosis: a meta-analysis. J Med Microbiol. 2015;64(7):702-7.

53. Hissen $\mathrm{AH}$, Wan AN, Warwas ML, Pinto $L$, Moore MM. The aspergillus fumigatus siderophore biosynthetic gene sidA, encoding L-ornithine N5oxygenase, is required for virulence. Infect Immun. 2005;73(9):5493-503.

54. Schrettl M, Bignell E, Kragl C, Joechl C, Rogers T, Arst HN Jr, et al. Siderophore biosynthesis but not reductive iron assimilation is essential for aspergillus fumigatus virulence. J Exp Med. 2004;200(9):1213-9.

55. Petrik M, Haas H, Dobrozemsky G, Lass-Florl C, Helbok A, Blatzer M, et al. 68Ga-siderophores for PET imaging of invasive pulmonary aspergillosis: proof of principle. J Nucl Med. 2010;51(4):639-45.

56. Waring P, Eichner RD, Mullbacher A, Sjaarda A. Gliotoxin induces apoptosis in macrophages unrelated to its antiphagocytic properties. J Biol Chem. 1988;263(34):18493-9.

57. Sutton $P$, Waring $P$, Mullbacher A. Exacerbation of invasive aspergillosis by the immunosuppressive fungal metabolite, gliotoxin. Immunol Cell Biol. 1996;74(4):318-22.

58. Sugui JA, Pardo J, Chang YC, Zarember KA, Nardone G, Galvez EM, et al. Gliotoxin is a virulence factor of aspergillus fumigatus: gliP deletion attenuates virulence in mice immunosuppressed with hydrocortisone. Eukaryot Cell. 2007;6(9):1562-9.

59. Amitani R, Taylor G, Elezis EN, Llewellyn-Jones C, Mitchell J, Kuze F, et al. Purification and characterization of factors produced by aspergillus fumigatus which affect human ciliated respiratory epithelium. Infect Immun. 1995;63(9):3266-71.

60. Bernardo PH, Brasch N, Chai CL. Waring P. A novel redox mechanism for the glutathione-dependent reversible uptake of a fungal toxin in cells. J Biol Chem. 2003;278(47):46549-55.

61. Domingo MP, Colmenarejo C, Martinez-Lostao L, Mullbacher A, Jarne C, Revillo MJ, et al. Bis(methyl)gliotoxin proves to be a more stable and reliable marker for invasive aspergillosis than gliotoxin and suitable for use in diagnosis. Diagn Microbiol Infect Dis. 2012;73(1):57-64.

62. Vidal-Garcia M, Domingo MP, De Rueda B, Roc L, Delgado MP, Revillo MJ, et al. Clinical validity of bis(methylthio)gliotoxin for the diagnosis of invasive aspergillosis. Appl Microbiol Biotechnol. 2016;100(5):2327-34.

63. Tuon FF. A systematic literature review on the diagnosis of invasive aspergillosis using polymerase chain reaction (PCR) from bronchoalveolar lavage clinical samples. Revista Iberoamericana de Micologia. 2007;24(2):89-94.

64. Sun W, Wang K, Gao W, Su X, Qian Q, Lu X, et al. Evaluation of PCR on bronchoalveolar lavage fluid for diagnosis of invasive aspergillosis: a bivariate metaanalysis and systematic review. PLoS One. 2011;6(12):e28467.

65. Avni T, Levy I, Sprecher H, Yahav D, Leibovici L, Paul M. Diagnostic accuracy of PCR alone compared to galactomannan in bronchoalveolar lavage fluid for diagnosis of invasive pulmonary aspergillosis: a systematic review. J Clin Microbiol. 2012;50(11):3652-8.

66. Arvanitis M, Ziakas PD, Zacharioudakis IM, Zervou FN, Caliendo AM, Mylonakis E. PCR in diagnosis of invasive aspergillosis: a meta-analysis of diagnostic performance. J Clin Microbiol. 2014;52(10):3731-42.
67. Cruciani M, Mengoli C, Loeffler J, Donnelly P, Barnes R, Jones BL, et al. Polymerase chain reaction blood tests for the diagnosis of invasive aspergillosis in immunocompromised people. Cochrane Database Syst Rev. 2015; https://doi.org/10.1002/14651858.CD009551.pub3.

68. Langridge PJ, Sheehan RL, Denning DW. Microbial yield from physiotherapy assisted sputum production in respiratory outpatients. BMC Pulmonary Med. 2016;16:23.

69. Baxter CG, Moore CB, Jones AM, Webb AK, Denning DW. IgE-mediated immune responses and airway detection of aspergillus and Candida in adult cystic fibrosis. Chest. 2013;143(5):1351-7.

70. European Society of Clinical Microbiology and Infectious Diseases (ESCMID)/ ESCMID Fungal Infection Study Group (EFISG) 2014 ESCMID Aspergillus guidelines. 24th European conference of clinical microbiology and infectious diseases (ECCMID). Spain: Barcelona; 2014. 10/05/2014.

71. Prattes J, Koidl C, Eigl S, Krause R, Hoenigl M. Bronchoalveolar lavage fluid sample pretreatment with Sputasol((R)) significantly reduces galactomannan levels. The Journal of infection. 2015;70(5):541-3.

\section{Ready to submit your research? Choose BMC and benefit from:}

- fast, convenient online submission

- thorough peer review by experienced researchers in your field

- rapid publication on acceptance

- support for research data, including large and complex data types

- gold Open Access which fosters wider collaboration and increased citations

- maximum visibility for your research: over $100 \mathrm{M}$ website views per year

At BMC, research is always in progress.

Learn more biomedcentral.com/submissions 